\title{
ANNULAR REFLECTORS FOR AN FEL RESONATOR
}

\author{
John F. WARD \\ Department of Physics, University of Michigan, Ann Arbor, Michigan 48109, USA
}

Scott A. VON LAVEN and Stephen B. SEGALL

KMS Fuston, Inc., PO Box 1567, Ann Arbor, Michigan 48106, USA

A resonator configuration suitable for a high-power, annular-mode FEL is discussed. The resonator is a four-element linear resonator with grazing-incidence reflectors. Cylindrically symmetric, paraboloid grazing incidence reflectors are used in order to take full advantage of the azimuthal polarization of the annular mode.

\section{Introduction}

To produce short-wavelength radiation from a lowenergy electron beam, KMS Fusion has proposed a two-stage FEL in which both FEL interactions take place in a cylindrical waveguide; nested sets of end mirrors provide feedback (fig. 1). An annular mode would be produced by an annular electron beam inside a helical wiggler in the first stage of the FEL [1]. As a result, the second-stage mode would also be annular and would have a large Rayleigh range in the interaction region. A second-stage resonator consisting of two almost-plane mirrors would thus seem appropriate, but such a resonator is inadequate in several respects. Since the power output of FELs may ultimately be limited by mirror damage thresholds, an important resonator characteristic is that the fraction of circulating power absorbed by unit area of the resonator mirrors should be small. Additional desirable characteristics are low sensitivity to mirror misalignment and short overall resonator length. It has been shown [2] that linear, four-element resonators with grazing incidence mirrors offer substantial advantages over almost-plane resonators in achieving these characteristics. We discuss here reso- nators of this type with azimuthally-symmetric paraboloid grazing-incidence mirrors which are appropriate for use with an annular mode and which also take advantage of the azimuthal polarization of the mode to achieve improved mirror reflectivity. Geometrical optics will be used to specify an approximately equivalent thin lens for the paraboloid reflector. The resonator analysis of ref. [2], which properly includes diffraction, will then be used to derive a specific resonator design.

\section{Paraboloid reflectors}

The field incident on the paraboloid can be approximated by the lowest-order annular free-space mode designated $\mathrm{TEM}_{01}^{*}$. The transverse distribution of the incident field, $E$, is given by

$|E|^{2}=\pi r^{2} \exp \left(-2 r^{2} / w^{2}\right)$.

The mode radius $w$ can be related to $\bar{r}$, the radius at maximum intensity, and $\hat{r}$, the half-power radius, defined such that half the power flows across the area bounded by $\hat{r}$. The relations are

$w=\sqrt{2} \bar{r}=1.3 \times \sqrt{2} \times \hat{r}$.

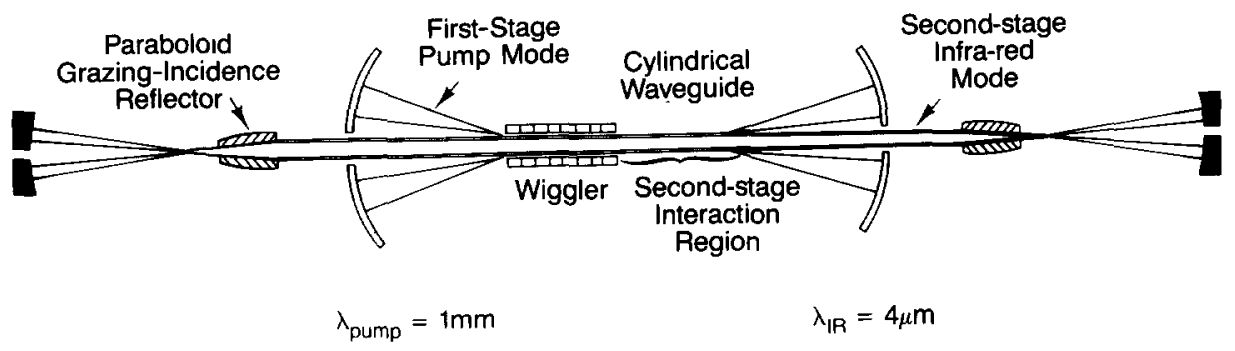

Fig. 1. Schematic diagram of a two-stage FEL proposed by KMS Fusion. 


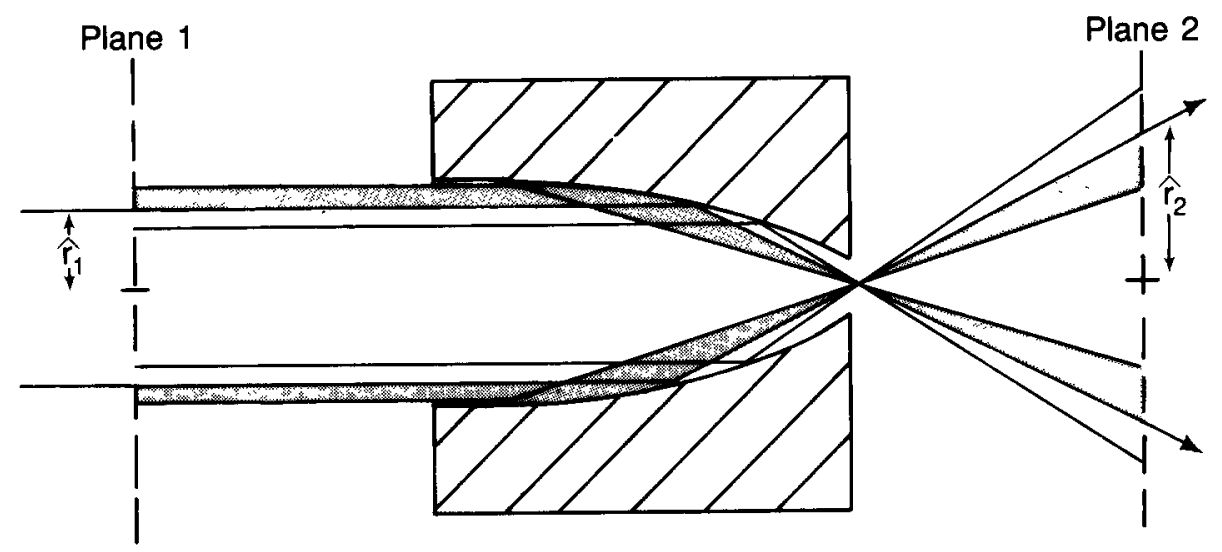

Fig. 2. Transformation of an annular mode by reflection from a paraboloid. The circle of radius $\hat{r}$ divides each plane into two regions each transmitting half the power.

Since the Rayleigh range is large in the interaction region, for this geometric-optics part of the discussion we use the approximation that $w$ is constant everywhere between the paraboloid reflectors.

The paraboloid reflector, symmetric about the resonator $z$-axis and with the vertex at $z=0$, is specified by

$r^{2}=4 a z$

where $a$ is the $z$ coordinate of the focus.

Fig. 2 illustrates the geometric-optics approach to transformation of the mode by reflection at the paraboloid. The field for the reflected mode is obtained by transferring the power each infinitesimal annular region of the incident mode at plane 1 to its geometrical image annulus in the reflected mode at plane 2 . One can see that the inner region of the mode at plane 1 transforms to the outer region of the mode at plane 2 and vice-versa. In particular, the outer half-power region of the incident mode bounded by $\hat{r}_{1}$ transforms into the inner half-power region of the reflected mode bounded by $\hat{r}_{2}$.

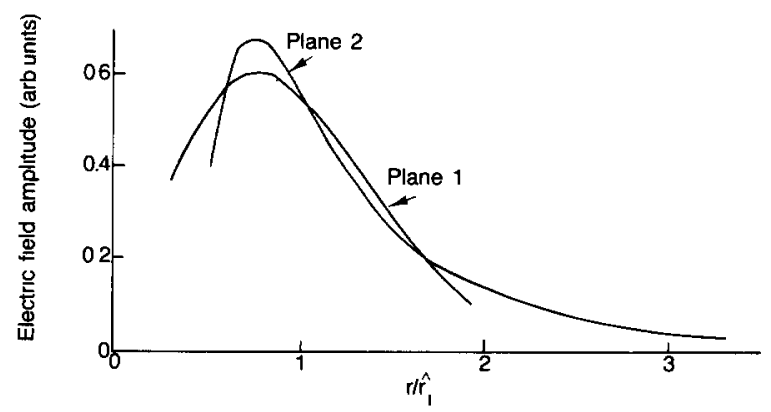

Fig. 3. Mode transformation by the paraboloid shown in fig. 2. A geometrical optics approximation has been used to transfer the power in each infinitesimal annulus at plane 1 to its image annulus at plane 2. The index $i$ used with $r$ indicates the plane.
Thus the incident ray defining $\hat{r}_{1}$ must reflect to form the ray defining $\hat{r}_{2}$. Using a value for $\hat{r}_{2}$ derived from $\hat{r}_{1}$ this way, we plot incident and reflected field amplitudes as a function of radii normalized to $\hat{r}$ in fig. 3. The reflected mode is similar in shape to the incident $\mathrm{TEM}_{01}^{*}$ mode with mode maxima occurring at the same value of $r / \hat{r}$. This geometric approach has heuristic appeal, and has been qualitatively confirmed by preliminary investigations with a numerical diffraction code.

The transformation demonstrated in fig. 3 gives one confidence to replace the paraboloid by an effective lens with effective focal length $f_{\mathrm{e}}$, given by

$f_{\mathrm{e}}=\left|\hat{r}^{2} / 4 a-a\right|$.

The paraxial focal length $a$ is regained by setting $\hat{r}=0$. For grazing incidence the focal point remains at $z=a$ but $z_{\mathrm{e}}$, the position of the equivalent thin lens, is given

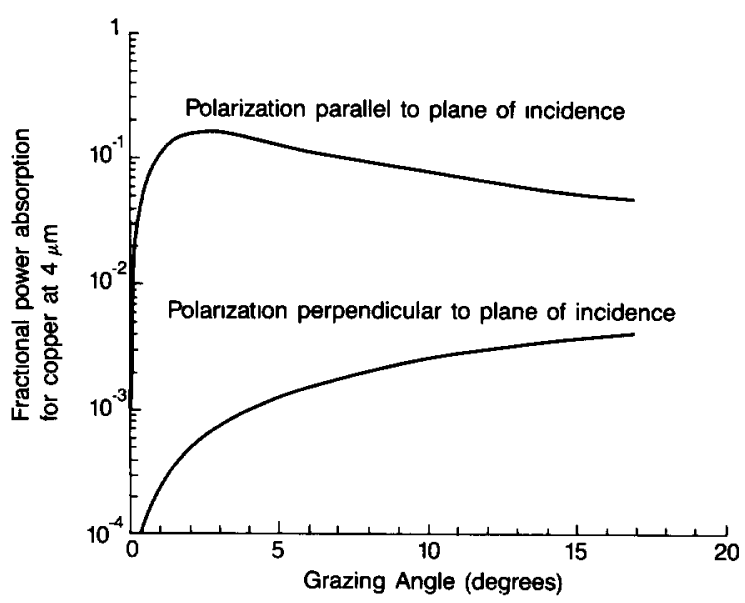

Fig. 4. Polarization-dependent fractional power absorption by copper at grazing incidence. 


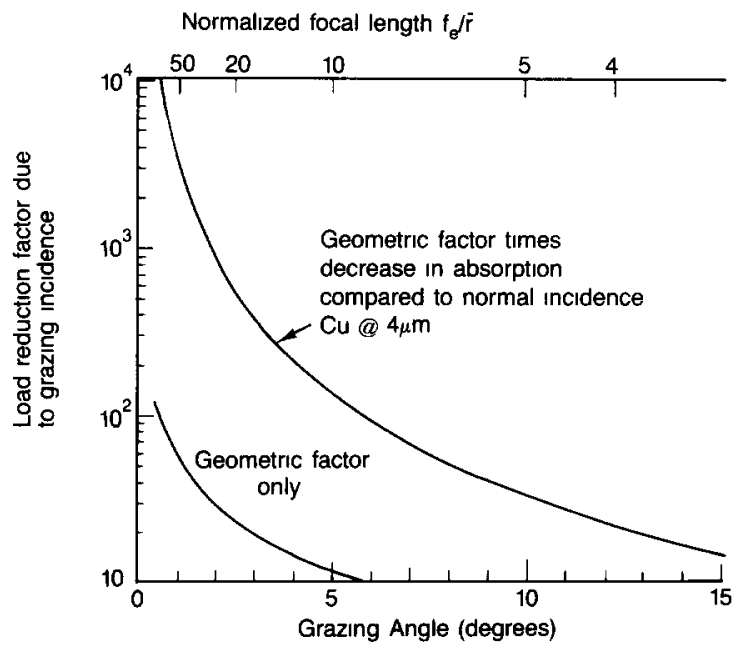

Fig. 5. Load reduction factor for a paraboloid reflector as a function of grazing incidence angle. The relation of normalized focal length to grazing incidence angle is also shown.

by

$z_{\mathrm{e}}=\hat{r}^{2} / 4 a$

The grazing incidence angle $\theta_{\mathrm{g}}$ evaluated at the mode intensity peak is

$\theta_{\mathrm{g}}=2 a / \bar{r}$.

Polarization-dependent fractional power absorption at grazing incidence for copper is shown in fig. 4 [3]. Azimuthal mode polarization and grazing incidence ensure low absorption loss. Geometrical distribution of the mode over a large mirror area further reduces absorption per unit area relative to circulating power in the resonator. We will refer to the absorption per unit area of mirror relative to that for a normal-incidence mirror located at the end of the interaction region, as the mirror load reduction factor. The two contributions to the load reduction are shown in fig. 5 as a function of grazing angle. The relationship between $\theta_{\mathrm{g}}$ and $f_{\mathrm{e}} \mathrm{im}$ plied by eqs. (4) and (6) is also displayed in fig. 5 .

\section{A specific resonator design}

Detailed parameters for the resonator shown in fig. 6 can now be computed. We consider the following specific set of values for illustration:

wavelength:

$4 \mu \mathrm{m}$,

length of interaction region:

$10 \mathrm{~m}$,

mode radius in the interaction

region $(w)$ :

$0.675 \mathrm{~cm}$

load reduction factor for paraboloid

reflectors:

1000

load reduction factor for end mirrors: 1000 .

A load reduction factor of 1000 at the end mirror requires that the mode radius there, $w_{\mathrm{m}}$, is

$w_{\mathrm{m}}=\sqrt{1000} \times w=21.5 \mathrm{~cm}$.

Fig. 5 shows that a load reduction factor of 1000 at the paraboloid reflectors requires:

$\theta_{\mathrm{g}}=1.9^{\circ}$,

$f_{\mathrm{e}}=25.5 \bar{r}=12.2 \mathrm{~cm}$.

Formulas from ref. [2] can now be used to find that the radii of curvature of the end mirrors are $3.9 \mathrm{~m}$ and that the separation of each end mirror from its corresponding effective lens is $3.9 \mathrm{~m}+f_{\mathrm{e}}+\Delta$, where $\Delta=(56 \pm 10)$ $\mu \mathrm{m}$. The $10 \mu \mathrm{m}$ length tolerance must be met to keep mode radii within about $10 \%$ of the values specified. Mirror misalignment tolerance is calculated to be 1.7 $\mu \mathrm{rad}$. This is the end-mirror misalignment which displaces the mode at the other end-mirror by one mode radius. End-mirror/end-mirror alignment is the most

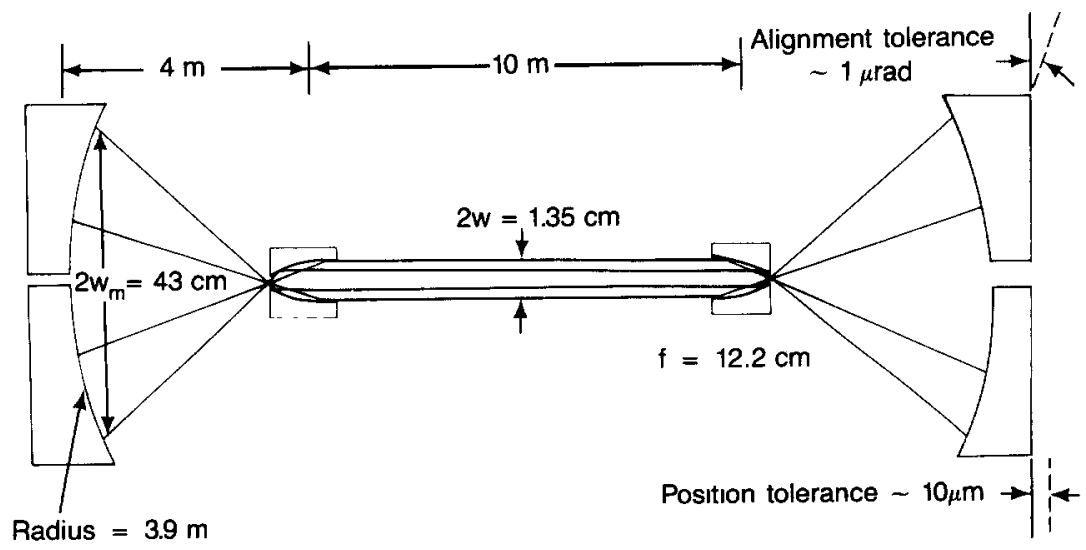

Fig. 6. Specific example of a resonator design using grazing incidence paraboloid mirrors. 
critical case. Mirror misalignment is discussed in further detail in ref. [2].

To specify the dimensions of the paraboloid, we assume that it is acceptable to truncate the paraboloid so that $1 \%$ of the power incident from the interaction region goes outside the paraboloid and another $1 \%$ passes through the vertex without reflection. These can be considered losses or used for external beam extraction. The minimum and maximum paraboloid radii are then $0.38 \bar{r}$ and $2.5 \bar{r}$ which in this example are $1.8 \mathrm{~mm}$ and $12 \mathrm{~mm}$. The corresponding length of the paraboloid is $48 \mathrm{~cm}$.

\section{Conclusion}

In conclusion, we have demonstrated that grazing incidence paraboloid reflectors could be used in a symmetric, linear, four-element resonator for a high-power annular-mode FEL. Mirror misalignment tolerance and length tolerance are restrictive but acceptable, mirror load reduction factors of 1000 are achieved at all mirrors, and the overall length of the device is less than 20 $m$. Further investigation with a numerical diffraction code is needed to refine resonator dimensions and to evaluate the effects of mode conversion at the paraboloid mirrors.

\section{Acknowledgement}

This work is sponsored by the US Office of Naval Research.

\section{References}

[1] S.B. Segall, H. Takeda, S. Von Laven, P. Diament and J.F. Ward, Free Electron Generators of Coherent Radiation, eds., Brau, Jacobs and Scully, Proc. SPIE 453 (1984) 178.

[2] S. Von Laven and J.F. Ward, IEEE J. Quantum Electron. QE-21 (1985) 1108.

[3] M. Born and E. Wolf, Principles of Optics, (Macmillan, New York, 1984) p. 628; AIP Handbook, 3rd ed. (McGraw-Hill, New York, 1972) p. 6. 